Raul Azevedo; Francisco Roberto de Azevedo; José Antônio de Lima;

Gilberto Barbosa Oliveira e Silva;

Ricardo Braga de Farias

\title{
Atratividade de iscas alimentares para insetos edáficos em vegetação de mata úmida da Floresta Nacional do Araripe na estação seca
}

\author{
Food baits atractiveness for edaphic insects in a humid forest from Floresta \\ Nacional do Araripe in the seasson dry
}

\author{
Raul Azevedo ${ }^{a}$ \\ Francisco Roberto de Azevedo ${ }^{b}$ \\ José Antônio de Lima ${ }^{c}$ \\ Gilberto Barbosa Oliveira e Silva ${ }^{d}$ \\ Ricardo Braga de Farias ${ }^{e}$
}

\footnotetext{
a Universidade Federal do Cariri; E-mail: raulbiologo@gmail.com

b Universidade Federal do Cariri; E-mail: roberto.azevedo@ufca.edu.br

c Universidade Federal do Cariri; E-mail: j.ceara@yahoo.com.br

d Universidade Federal do Cariri; E-mail: gilbarbosa musico@hotmail.com

e Universidade Federal do Cariri; E-mail: ribrava@live.com
}

Recebido em: 16/08/2017 I Aceito em: 11/05/2018

\section{ARTIGO}

\section{RESUMO}

Este estudo objetivou analisar preliminarmente a atratividade de diferentes iscas alimentares para os insetos edáficos presentes na Mata Úmida da Floresta Nacional do Araripe. Para isso, foram instaladas 24 armadilhas de solo, no Crato-CE, no período de 20 de setembro a 29 de novembro de 2013, avaliando-se iscas de carne de frango em putrefação, esterco de suíno fresco, sardinha em conserva, banana fermentada, etanol, além da testemunha (armadilha sem isca). As coletas dos insetos nas armadilhas foram feitas semanalmente, substituindo-se as iscas alimentares, as soluções de água e detergente e os insetos capturados foram levados ao Laboratório de Entomologia Agrícola da Universidade Federal do Cariri, Campus do Crato, para triagem e identificação em nível de ordem e família. As iscas que atraem uma maior diversidade de insetos edáficos são as de esterco suíno 
fresco e as de banana em fermentação. Em baixa temperatura e alta umidade a isca de etanol demonstra boa eficiência na captura de Scolytidae. Nas condições da Mata Úmida e do Cariri cearense a isca de frango apresenta baixa eficiência na captura de insetos edáficos. Foi observada uma riqueza de dez famílias pertencentes a seis ordens, sendo poucas dominantes em termos de abundância de indivíduos, diferente do que seria esperado na estação seca. Embora restritos espacial e temporalmente, os resultados deste trabalho possibilitaram observar a atratividade de várias iscas e a preferência alimentar de algumas famílias de insetos. No entanto, novas amostragens durante períodos mais longos precisam ser realizadas com o objetivo de capturar um maior número de indivíduos e, assim, obter maior representatividade das famílias a fim de alcançar resultados mais confiáveis.

Palavras-chave: Captura. Insecta. Odores. Armadilhas de queda.

\section{ABSTRACT}

This study aimed to analyze previously the attractiveness of differences food baits by edaphic insect's presents in Rainforest ecosystem of the Floresta Nacional do Araripe. For this, 24 pitfall traps had been installed in Crato, from $20^{\mathrm{TH}}$ September to $29^{\mathrm{TH}}$ November 2013, evaluating rot chicken bait, fresh suine manure bait, canned sardines bait, barmy banana bait, alcoholic bait and control (trap without bait). The collects of insects were weekly, changing the baits and water detergent solution and the insects collected were been transported to Entomology Laboratory of Universidade Federal do Cariri, Campus Crato, to identification in level of order and family. The baits that best attracted more insect's diversity are fresh suine manure and barmy banana. At low temperature and high humidity the ethanol bait exhibit good efficiency in the capture of Scolytidae. In the conditions of Rainforest and the Cariri of Ceará, chicken bait exhibit low efficiency in the capture of edaphic insects. It was observed a richness of 10 families belonged to six orders, few families were dominant in abundance of individuals, opposite of would be expected. Although the results of this work would be temporal and space restricted, it was possible to observe the attractiveness of some baits and the feed habits of some family. However, new samples using long periods must be realized aiming capture a bigger number of individuals and, therefore, to find a bigger representatively of the families in order to get more confinable results.

Key Words: Capture. Insecta. Smell. Pitfall traps. 
Raul Azevedo; Francisco Roberto de Azevedo; José Antônio de Lima;

Gilberto Barbosa Oliveira e Silva;

Ricardo Braga de Farias

\section{INTRODUÇÃO}

Os inventários e a identificação de insetos nos ecossistemas permitem as prevenções e/ou remediações de impactos nos diferentes ambientes, caracterizando a importância de estudos que identifiquem grupos de indicadores ecológicos potenciais. Dessa forma, são demonstradas as respostas das comunidades de insetos frente às perturbações ambientais (WINK et al., 2005; SILVEIRA et al., 2010).

Os insetos edáficos possuem hábitos alimentares diversos, apresentando diferentes adaptações fisiológicas para a captura, digestão e localização do alimento (GILLOTT, 2005). Estes podem ser predadores especialistas ou generalistas e, por sua vez, potencializam a sua capacidade de explorar recursos alimentares, alternando as fontes de alimento de acordo com sua conveniência (SCHOWALTER, 2006; BEGON; TOWNSEND; HARPER, 2009). A preferência alimentar reduz a competição intra e interespecífica entre espécies de insetos, permitindo que exista uma gama de espécies habitando um ecossistema (DA SILVA; AUDINO, 2011). As iscas atrativas tais como carne fresca e frutas em putrefação, podem ser utilizadas como mecanismos para atração de grupos específicos de insetos, como as formigas cortadeiras e os besouros que vivem no solo (SILVA; DO AMARAL, 2013).

Armadilhas de queda (pitfalls trap) são usadas para amostrar tanto animais vertebrados como invertebrados. Dentre estes estão os artrópodes ativos em solo e serrapilheira, que possibilitam a realização de estudos sobre a diversidade, flutuação populacional e distribuição anual de espécies (MOLDENKE, 1994; BARBOSA, 2008; MOREIRA; HUISING; BIGNELL, 2010). Essas armadilhas são úteis para mensuração da diversidade e para inventários de fauna, onde o número de espécies coletadas é proporcional ao número de armadilhas instaladas e de áreas amostradas (MOREIRA; HUISING; BIGNELL, 2010; SILVA; DO AMARAL, 2013).

$A$ armadilha consiste em um recipiente plástico (partindo de um simples copo de café ou balde) que é enterrado no nível do solo. No seu interior é colocada uma solução conservante e algumas gotas de detergente neutro para quebrar a tensão superficial da solução. Os artrópodes ao se deslocarem, caem nos pitfall trap e ao entrarem em contato com a solução, descem para o fundo do pote, vindos a óbito, 
porém mantendo-se preservados devido a solução conservante (ALMEIDA; RIBEIRO-COSTA; MARINONI, 2003).

Esta solução conservante pode ser álcool a 70\%, ou até mesmo uma solução composta de álcool a $70 \%$ e formaldeído a $4 \%$ e pode ser também uma solução salina supersaturada, (ALMEIDA; RIBEIRO-COSTA; MARINONI, 2003; NOGUEIRAPARANHOS; CARVALHO; LIMA, 2015).

Essas armadilhas têm sido consideradas eficientes em diferentes ecossistemas tais como Floresta Amazônica, Savanas Africanas e Cerrados (PARR; CHOWN, 2001; LOPES; VASCONCELOS, 2008; PEIXOTO et al., 2010; SOUZA et al., 2012). Tal eficácia é atribuída principalmente ao tempo que a armadilha permanece em operação na área de estudo e ao uso facultativo de iscas como atrativos alimentares (BESTELMEYER et al., 2000; ALMEIDA et al., 2003 ou ALMEIDA; RIBEIRO-COSTA; MARINONI, 2003). Contudo, seu uso é visto com parcimônia, pois tornam a amostra tendenciosa a grupos específicos em virtude de apresentarem maior capacidade olfativa (MOREIRA; HUISING; BIGNELL, 2010).

A depender do grupo taxonômico a ser amostrado e do tipo de dieta observado para o táxon (i.e., predadores especialistas ou generalistas), seleciona-se o tipo de isca desejado. Para amostragem de formigas generalistas ou dominantes no ambiente utiliza-se isca a base de carboidrato, como pão e mel (SARMIENTO, 2003) ou proteína animal, como sardinha (VALE JÚNIOR et al., 2017). Para besouros como rola-bosta (Coleoptera: Scarabaeidae), pode-se utilizar iscas a base de fezes humanas e de animais de pecuária; frutas e legumes; carne e miúdos de animais apodrecidos; ovos de galinha; insetos mortos e fungos Basidiomycetes em decomposição (DA SILVA; AUDINO, 2011). Para besouros carabídeos podem-se utilizar iscas como carne, fezes e bananas (QUINTEIRO; LOPES; MARTINS, 2012), que embora esses besouros sejam carnívoros, as iscas utilizadas atraem insetos que constituem a dieta alimentar dos carabídeos.

O objetivo desta pesquisa foi avaliar o uso de diferentes tipos de iscas alimentares para amostragem de insetos edáficos em uma área de Mata Úmida da Floresta Nacional do Araripe, Estado do Ceará, durante a estação seca. Essas informações são úteis em programas de manejo da FLONA e na conservação da biodiversidade de insetos da Chapada do Araripe. 
Raul Azevedo; Francisco Roberto de Azevedo; José Antônio de Lima;

Gilberto Barbosa Oliveira e Silva;

Ricardo Braga de Farias

\section{MATERIAIS E MÉTODOS}

A pesquisa foi realizada na Floresta Nacional do Araripe - FLONA Araripe ( $7^{\circ}$ $15^{\prime} 18,82^{\prime \prime}$ S, $39^{\circ} 29^{\prime} 31,95^{\prime \prime}$ W), em sua parte localizada na região do Cariri cearense, município do Crato, no período de 20 de setembro a 29 de novembro de 2013.

Os tratamentos foram representados por iscas alimentares constituídas por carne de frango em putrefação, esterco de suíno fresco, sardinha em conserva, banana em processo de fermentação, etanol, além da testemunha (armadilha sem isca). Todos distribuídos em quatro repetições, totalizando 24 unidades amostrais constituídas de armadilhas de solo acopladas aos pitfall traps constituídas de garrafas PET.

Semanalmente, o conteúdo de cada armadilha foi recolhido e as iscas foram trocadas. Além disso, no interior de cada garrafa, foi adicionado $250 \mathrm{~mL}$ de uma solução de água + detergente neutro na proporção de 3:1 para quebrar a tensão superficial da água. As armadilhas foram dispostas em quatro linhas paralelas, contendo seis armadilhas em cada linha, com espaçamentos de 30 metros nas linhas e entre elas, obtendo-se uma área efetiva de $13.500 \mathrm{~m}^{2}$.

Os insetos coletados foram acondicionados em recipientes plásticos de 100 $\mathrm{mL}$ contendo álcool a 70\% e, em seguida, levados ao Laboratório de Entomologia Agrícola da Universidade Federal do Cariri, Campus do Crato, para triagem e identificação em nível de ordem e família, utilizando-se chaves ilustradas de identificação (BUZZI, 2008; TRIPLEHORN; JOHNSON, 2011).

A curva de rarefação é uma ferramenta que serve para mensurar taxa de captura de espécies/indivíduos ao longo das unidades amostrais (MAGURRAN, 2004), ressaltando-se que o esforço amostral foi suficiente. As curvas de rarefação bem como a atratividade das iscas foram avaliadas por meio de Anova-I, utilizandose para tal o programa PAST (HAMMER et al, 2001). Os dados meteorológicos foram obtidos pela plataforma do Instituto Nacional de Meteorologia - INMET (INMET, 2013). 


\section{RESULTADOS}

Foi coletado um total de 10.083 insetos edáficos, distribuídos em seis ordens e dez famílias, conforme apresentado na Tabela 1. Formicidae foi a família mais abundante, seguida de Scolytidae. A atratividade foi mensurada por meio de uma curva de rarefação, e por meio dessa, ficou evidenciado que o esterco de suíno fresco exibiu maior tendência à estabilização, decorrente de um maior número de insetos coletados, seguido da banana em fermentação (Fig. 1).

Tabela 1. Número total de ordens, famílias e espécimes de insetos coletados nas armadilhas de solo contendo iscas alimentares, realizadas na Mata Úmida da Floresta Nacional do Araripe, Crato-CE, na estação seca de 2013.

\section{Iscas}

\begin{tabular}{llccccccc}
\hline Ordens & Famílias & Sem isca & Frango & Esterco & Sardinha & Banana & Etanol & Total \\
\hline Coleoptera & Scolytidae & 408 & 161 & 875 & 75 & 552 & 498 & $\mathbf{2 . 5 6 9}$ \\
& Nitidulidae & 72 & 9 & 201 & 57 & 21 & 64 & $\mathbf{4 2 4}$ \\
& Curculionidae & 13 & 16 & 27 & 7 & 11 & 20 & $\mathbf{9 4}$ \\
& Staphylinidae & 3 & 6 & 16 & 2 & 6 & 3 & $\mathbf{3 6}$ \\
& Scarabaeidae & 9 & 2 & 0 & 1 & 0 & 1 & $\mathbf{1 3}$ \\
\hline Hemiptera & Cydnidae & 200 & 252 & 335 & 108 & 360 & 107 & $\mathbf{1 . 3 6 2}$ \\
\hline Orthoptera & Gryllidae & 145 & 140 & 382 & 118 & 175 & 197 & $\mathbf{1 . 1 5 7}$ \\
\hline Blattodea & Blatidae & 81 & 258 & 412 & 170 & 557 & 87 & $\mathbf{1 . 5 6 5}$ \\
\hline Hymenoptera & Formicidae & 319 & 412 & 721 & 424 & 589 & 349 & $\mathbf{2 . 8 1 4}$ \\
\hline Dermaptera & Forficulidae & 4 & 6 & 12 & 2 & 12 & 13 & $\mathbf{4 9}$ \\
\hline Total & & $\mathbf{1 . 2 5 4}$ & $\mathbf{1 . 2 6 2}$ & $\mathbf{2 . 9 8 1}$ & $\mathbf{9 6 4}$ & $\mathbf{2 . 2 8 3}$ & $\mathbf{1 . 3 3 9}$ & \\
\hline Total Geral & & & & & & & & $\mathbf{1 0 . 0 8 3}$
\end{tabular}


Raul Azevedo; Francisco Roberto de Azevedo; José Antônio de Lima;

Gilberto Barbosa Oliveira e Silva;

Ricardo Braga de Farias

Figura 1: Rarefação das iscas utilizadas como atrativo alimentar.

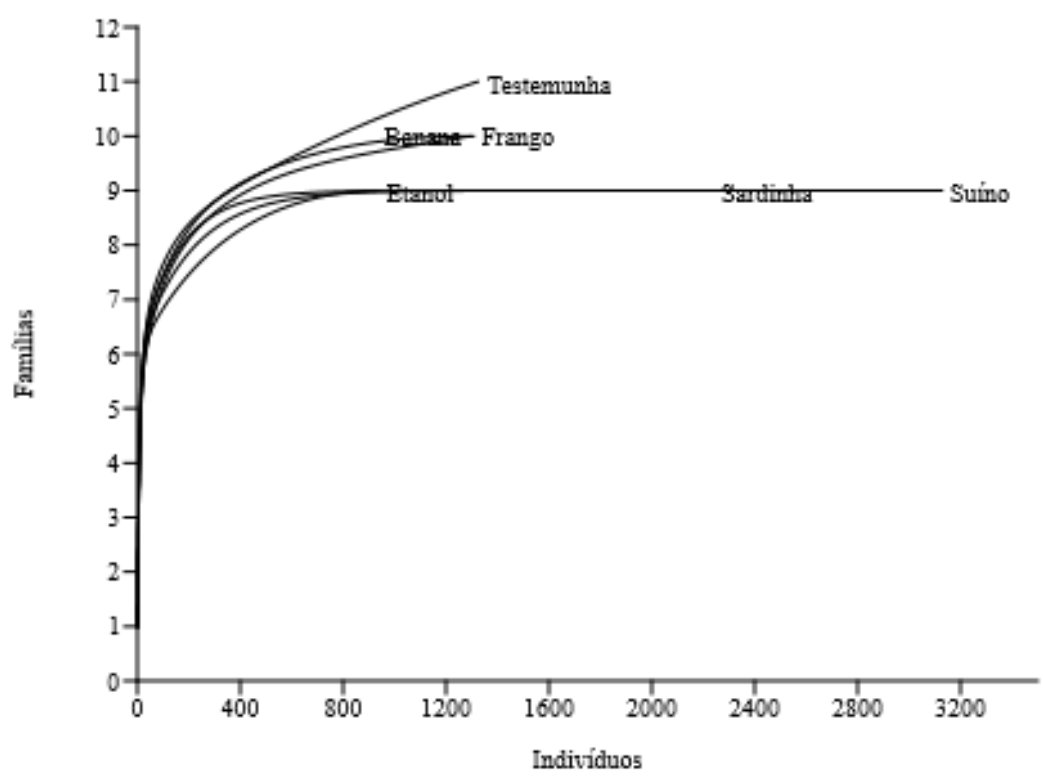

A atratividade das iscas não foi significativa para as famílias exceto para Gryllidae, Blattidae e Scolytidae (Tabela 2).

Tabela 2: Atratividade das iscas frente às famílias coletadas.

\begin{tabular}{|c|c|c|c|c|c|c|}
\hline Família & Frango & Banana & Excremento Suíno & Etanol & Sardinha & Controle \\
\hline Cydinidae & NS & NS & NS & NS & NS & NS \\
\hline Grillidae & NS & SIG & SIG & NS & NS & NS \\
\hline Blattidae & NS & NS & NS & NS & SIG & SIG \\
\hline Formicidae & NS & NS & NS & NS & NS & NS \\
\hline Nitidulidade & NS & NS & NS & NS & NS & NS \\
\hline Scolytidae & NS & SIG & SIG & SIG & SIG & NS \\
\hline Forficulidae & NS & NS & NS & NS & NS & NS \\
\hline Staphilidae & NS & NS & NS & NS & NS & NS \\
\hline Curculionidae & NS & NS & NS & NS & NS & NS \\
\hline Scarabaeidae & NS & NS & NS & NS & NS & NS \\
\hline
\end{tabular}

Ciência e Sustentabilidade - CeS / Juazeiro do Norte, v. 4, n. 1, p. 22-38, jan/jun - 2018 
Atratividade de iscas alimentares para insetos edáficos em vegetação de mata úmida da

Floresta Nacional do Araripe na estação seca

Os valores de precipitação, temperatura e pluviosidade são apresentados na Tabela 3. Observa-se que não houve precipitação durante o período e que a média da temperatura nos dias das coletas foi de $28^{\circ} \mathrm{C}$, enquanto que a média da umidade relativa do ar foi de 51\%. A flutuação populacional das famílias coletadas é evidenciada na Figura 2. Observa-se que todas as iscas exibiram tendências distintas a redução da taxa de captura dos insetos em função do aumento da temperatura.

Figura 2: Flutuação populacional das famílias mais abundantes durante a estação seca de 2013. Legenda: A - Cydnidae; B - Gryllidae; C - Scolytidae; D - Formicidae; E - Blattidae.

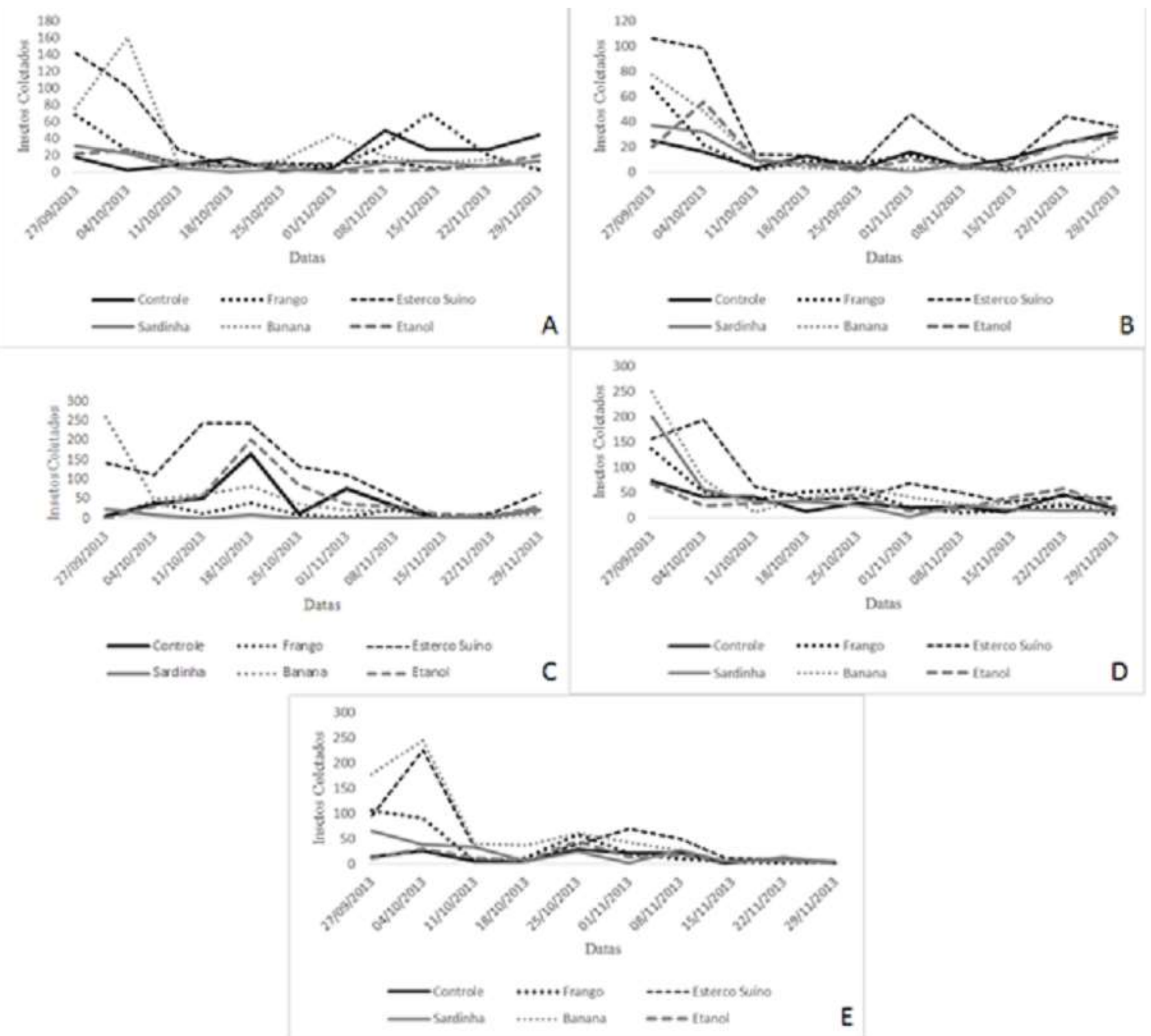

Ciência e Sustentabilidade - CeS / Juazeiro do Norte, v. 4, n. 1, p. 22-38, jan/jun - 2018 
Raul Azevedo; Francisco Roberto de Azevedo; José Antônio de Lima;

Gilberto Barbosa Oliveira e Silva;

Ricardo Braga de Farias

Tabela 3: Dados meteorológicos do Município do Crato-CE, em 2013. Informações obtidas pelo Instituto Nacional de Meteorologia - INMET.

\begin{tabular}{cccc}
\hline Datas & Precipitação & Temperatura Média & Umidade Relativa \\
\hline $27 / 09 / 2013$ & 0 & 29,85 & 48,25 \\
$04 / 10 / 2013$ & 0 & 27,85 & 46,66 \\
$11 / 10 / 2013$ & 0 & 27,1 & 43,66 \\
$18 / 10 / 2013$ & 0 & 28,75 & 53,00 \\
$25 / 10 / 2013$ & 0 & 28,55 & 57,66 \\
$01 / 11 / 2013$ & 0 & 27,15 & 46,33 \\
$08 / 11 / 2013$ & 0 & 28,2 & 47,66 \\
$14 / 11 / 2013$ & 0 & 29,4 & 53,33 \\
$22 / 11 / 2013$ & 0 & 27,15 & 55,66 \\
$29 / 11 / 2013$ & 0 & 28,4 & 57,66 \\
\hline Média & $\mathbf{0}$ & $\mathbf{2 8 , 2 4}$ & $\mathbf{5 0 , 9 8}$ \\
\hline
\end{tabular}

\section{DISCUSSÃO}

Formicidae foi a família que apresentou maior número de indivíduos capturados. Isso se deve ao fato de que as formigas podem ser carnívoras (alimentando-se de carne de outros animais vivos ou mortos), fitófagas, outras alimentam-se de fungos e muitas se alimentam de seiva, néctar e mela exsudada de outros insetos como pulgões e cochonilhas (TRIPLEHORN; JOHNSON, 2011).

A variedade de iscas ofertadas permitiu que diversas guildas de formigas fossem amostradas, justificando assim o elevado número de indivíduos capturados. A melhor taxa de captura, representada pelo dia 27/09/2013 (Fig. 2), corresponde ao início das coletas. Este fato pode ser atribuído a temperatura (KASPARI, 2000) que provavelmente favoreceu a liberação de odores das iscas, dentre elas a banana e a sardinha, atraindo assim guildas tanto frugívoras quanto carnívoras. A atratividade de tais iscas é descrita e recomendada no protocolo elaborado por Bestelmeyer et al. (2000) e seguido internacionalmente como forma de padronizar amostragens de formigas.

Os escolitídeos são besouros conhecidos por abrirem galerias no interior das cascas e no interior das árvores, atacando árvores doentes, estressadas ou madeiras recém-cortadas (MARINONI et al., 2001). Apesar da isca de esterco ter Ciência e Sustentabilidade - CeS / Juazeiro do Norte, v. 4, n. 1, p. 22-38, jan/jun - 2018 
capturado mais indivíduos da família Scolytidae, a isca etanólica é a que apresenta melhores valores de captura (Fig. 2-C) desses indivíduos (ROCHA, 2010). Em nosso estudo, a isca etanólica obteve a segunda maior taxa de captura, podendo ser atribuída à grande quantidade de árvores em decomposição ou mortas, as quais poderiam atrair mais a atenção dos espécimes, afastando-os das armadilhas. Isso é um indicador ecológico das condições ambientais da Mata Úmida da FLONA. Tal informação sinaliza ações que devem ser tomadas para se manter a sustentabilidade desse ecossistema no Cariri Cearense para as condições semiáridas.

Excrementos de animais são recursos alimentares que concentram grande quantidade de energia e, por isso, são utilizados por vários grupos de artrópodes, entre eles muitos besouros (MEDRI; LOPES, 2001). Insetos coprófagos e necrófagos podem ser atraídos por fezes humanas e de diversos animais (AQUINO et al., 2006). Dessa forma, os trabalhos cuja metodologia se baseia na combinação armadilha de solo e iscas contendo fezes têm sido mais eficientes para formigas e besouros.

Korasaki et al. (2006) constataram que fezes suínas e bananas fermentadas demonstraram baixa atratividade para besouros da família Scarabaeidae. Esses resultados estão de acordo com os encontrados nesse trabalho, pois foram capturados apenas 13 espécimes dessa família. Esse padrão diverge do observado para Nitidulidae ao se utilizar o esterco suíno por apresentar o maior número de indivíduos capturados. Os besouros nitidulídeos geralmente são encontrados próximos a locais que contenham líquidos em fermentação ou em locais com melhor estado de preservação (TRIPLEHORN; JOHNSON, 2011; LESCHEN; MARRIS, 2012).

A baixa atratividade dentro da família Scarabaeidae pode ser atribuída à alta generalidade alimentar (LARSEN et al., 2006), fazendo com que muitos desses besouros utilizem outros recursos em estado semelhante de decomposição (HANSKI; CAMBEFORT, 1991). Entretanto, um estudo realizado com a família Carabidae em áreas florestadas com diferentes níveis de degradação mostrou que a isca contendo carne apresenta maior taxa de captura de indivíduos e espécies, 
Raul Azevedo; Francisco Roberto de Azevedo;

José Antônio de Lima;

Gilberto Barbosa Oliveira e Silva;

Ricardo Braga de Farias

enquanto que iscas de banana e fezes apresentam valores similares entre si para abundância e riqueza de espécies (QUINTEIRO; LOPES; MARTINS, 2012).

Milhomem, De Mello e Diniz (2003) analisaram três fitofisionomias de Cerrado e observaram que existem diferenças significativas da diversidade, ou seja, a associação entre riqueza de espécies e abundância relativa das mesmas, conforme Magurran (2004), de besouros escarabeídeos Sensu Strictu coletados por meio de pitfalls traps, usando iscas de carcaça e fezes.

A família Curculionidae apesar de serem classificadas como dominantes e muito abundantes na Floresta Nacional do Araripe (AZEVEDO et al., 2011), apresentaram valores intermediários de abundância quando comparados as demais famílias de coleópteros coletadas no presente trabalho. $\mathrm{O}$ baixo número de indivíduos associados a cada tipo de isca pode ser atribuído ao hábito alimentar endofitófago (OBERPRIELER et al., 2007).

A presença de um maior número de indivíduos de Staphynilidae associada ao esterco suíno sugere uma maior diversidade de insetos que venham a se alimentar desta isca. A literatura reporta que os estafilinídeos são predadores em fezes bovinas devido a diversidade de espécies existentes e a uma maior riqueza de indivíduos (MARCHIORI; DE OLIVEIRA; LINHARES, 2000), sendo considerados predadores de dípteros associados a fezes de bovinos (GUIMARÃES; MENDES, 1998).

Apesar das iscas à base de proteína animal (frango e sardinha) exibirem os menores valores de indivíduos coletados, a literatura forense reporta a ocorrência de 41 espécies de insetos pertencentes a oito ordens que foram associadas a carcaças de porco Sus scrofa Linnaeus 1758, em estudo realizado em fragmento de Mata de Araucária (PANIGALLI; SOLIGO, 2013).

De acordo com a literatura forense, esperava-se encontrar indivíduos pertencentes a família Silphidae, visto que esta é uma das principais famílias necrófagas, juntamente com Cleridae e Dermestidae (OLIVEIRA-COSTA, 2007).

A isca de banana capturou mais percevejos castanhos no dia 04/09/2013, obtendo-se 161 espécimes, enquanto que o esterco de suíno capturou no dia 27/09/2013, 142 espécimes, decrescendo as capturas em seguida. Nessas datas, essas iscas utilizadas foram as mais eficientes quando comparadas com a armadilha sem isca e as demais, enquanto que a isca de etanol apresentou comportamento Ciência e Sustentabilidade - CeS / Juazeiro do Norte, v. 4, n. 1, p. 22-38, jan/jun - 2018 
semelhante à testemunha (sem isca). Nesses dias a temperatura média ficou próxima dos $30^{\circ} \mathrm{C}$ e ocorreu baixa umidade relativa do ar. Tais condições meteorológicas podem ter favorecido uma maior fermentação da banana e a decomposição do esterco promovendo maior atratividade para os insetos dessa família, ocorrendo o contrário para o etanol que certamente volatilizou-se mais rapidamente.

A isca de esterco suíno capturou mais grilos no dia 27/09/2013, obtendo-se 106 espécimes no total, enquanto que a isca de banana capturou 77 espécimes. Em seguida, houve redução na captura utilizando a isca de banana, enquanto que na de esterco suíno ocorreram oscilações ao longo das avaliações.

A Mata Úmida, por apresentar um ambiente fechado e com temperaturas amenas, certamente propiciou condições ideais para os grilos, graças a maior concentração de serrapilheira e de matéria orgânica. A fauna desses insetos é pobre em espécies e sua abundância é relativamente baixa em fragmentos de florestas (SPERBER, 1999), de modo que tal abundância encontrada pode ter sido em decorrência de perturbações físicas, visto que estas causam respostas rápidas na comunidade de grilos, podendo ser indicadores em uma escala local (SPERBER et al., 2007).

Contudo, em um inventário mais recente da entomofauna da Floresta Nacional do Araripe, a família Gryllidae (Orthoptera) foi considerada a de maior frequência entre todas as famílias coletadas para área de Mata Úmida (AZEVEDO et al., 2011), bem como dominante e muito abundante, baseado nos parâmetros definidos por Silveira-Neto et al. (1976). Do mesmo modo, os valores obtidos para Blattidae (Blattodea) contrasta com os valores observados por Azevedo et al. (2011), onde as baratas foram consideradas não dominantes e com baixa frequência, e em nosso estudo as mesmas obtiveram terceiro maior número de indivíduos coletados.

As iscas que atraem uma maior diversidade de insetos edáficos são as de esterco suíno fresco e a de banana em fermentação. A temperatura também mostrou ser um dos fatores que influenciam a captura de insetos concomitante com outros fatores, como a umidade e o tipo de isca utilizado, potencializando assim a captura de escolitídeos. 
Raul Azevedo; Francisco Roberto de Azevedo; José Antônio de Lima;

Gilberto Barbosa Oliveira e Silva;

Ricardo Braga de Farias

\section{CONSIDERAÇÕES FINAIS}

Nas condições da estação seca da Mata Úmida e do Cariri cearense a isca de frango apresenta baixa eficiência na captura de insetos edáficos. Além disso, foi observada uma parcela da diversidade de famílias observadas, em que poucas foram dominantes em termos de abundância de indivíduos, como seria esperado na estação seca.

Embora restritos espacial e temporalmente, os resultados possibilitaram observar a atratividade de várias iscas e a preferência alimentar de algumas famílias insetos. No entanto, novas amostragens durante períodos mais longos, com maior área de abrangência e maior tempo de operação precisam ser realizadas com o objetivo de capturar um maior número de indivíduos e, assim, obter maior representatividade das famílias que não foram amostradas no presente estudo.

Esse trabalho contribui para um melhor conhecimento da fauna de insetos edáficos e da sua dinâmica, bem como a preservação da Floresta Nacional do Araripe.

\section{Agradecimentos}

Ao Chefe da FLONA, William Brito pela autorização e aporte para o desenvolvimento dessa pesquisa.

\section{REFERÊNCIAS}

ALMEIDA, L.M.; RIBEIRO-COSTA, C.S.; MARINONI, L. Manual de coleta, conservação, montagem e identificação de insetos. Ribeirão Preto: Editora Holos, 78p. 2003.

AQUINO, A.M.; AGUIAR-MENEZES, E.L..; QUEIROZ, J.M. Recomendações para coleta de artrópodes terrestres por armadilhas de queda ("pitfall-traps"). Seropédica, Embrapa Agrobiologia, p.1-8, 2006.

ARAÚJO, C.C.; NOMELINI, Q.S.S.; PEREIRA, J.M.; LIPORACCI, H.S.N.;

KATAGUIRI, V. S. Comparação da abundância de invertebrados de solo por meio da estimação intervalar encontrados em diferentes ambientes na cidade de Ituiutaba MG. Bioscience Journal, v.26, n.5, p. 817-823, 2010.

Ciência e Sustentabilidade - CeS / Juazeiro do Norte, v. 4, n. 1, p. 22-38, jan/jun - 2018 
AZEVEDO, F.R.; De MOURA, M.A.R.; ARRAIS, M.S.B.; NERE, D.R. Composição da entomofauna da Floresta Nacional do Araripe em diferentes vegetações e estações do ano. Revista Ceres, Viçosa, v.58, n. 6, p.740-748, 2011.

BARBOSA, O. de A. A. Entomofauna de solo em áreas de vegetação nativa e de cultivo de cana-de-açúcar no município de União Piauí. 2008. 96f. Dissertação (Mestrado em Agronomia). Universidade Federal de Piauí, Teresina, 2008.

BESTELMEYER, B.T.; AGOSTI, D.; ALONSO, L.E.; BRANDÃO, C.R.F.; BROWN JR., W.L.; DELABIE, J.H.C.; SILVESTRE, R. Field techniques for the study of Ground-Dwelling ants: an overview, description and evaluation. In: AGOSTI, D.; MAJER, J.D.; ALONSO, L.E.; SCHULTZ, T.R. (Eds.); Ants: standard methods for measuring and monitoring biodiversity. Washington and London: Smithsonian Institution Press, p.122-269. 2000.

BEGON, M; TOWNSEND, C.R.; HARPER, J.L. Ecologia: de indivíduos a ecossistemas. Porto Alegre: Artmed Editora, 719p. 2009.

BUZZI, Z.J. Entomologia didática. 4ª ed. Curitiba: Editora UFPR, 579p. 2008.

GILLOTT, C. Entomology. Dordrecht: Springer, 831p. 2005.

GUIMARÃES, J.A.; MENDES, J. Succession and abundance of Staphylinidae in cattle dung in Uberlândia, Brazil. Memórias do Instituto Oswaldo Cruz, v.93, p.127-130, 1998.

HAMMER, O; HARPER, D.A.T.; RYAN, P.D. PAST: Paleontological statistics software package for education and data analyses. Paleontologia eletronica 4. 2001.

HANSKI, I.; CAMBEFORT, Y. Competition in dung beetles. In: HANSKI, I.;

CAMBEFORT, Y. (Eds.). Dung beetle ecology. Princeton, Princeton University Press. p.305-329. 1991.

INMET. Dados meteorológicos - Crato. 2013. Disponível em: <http://www.agritempo.gov.br/agroclima/pesquisa> Acesso em: 06 de dezembro de 2013.

KASPARI, M. A primer on ant ecology. In: AGOSTI, D.; MAJER, J.D.; ALONSO, L.E.; SCHULTZ, T.R. (Eds.). Ants: standard methods for measuring and monitoring biodiversity. Washington and London: Smithsonian Institution Press. p. 9-24. 2000.

KORASAKI, V.; BROWN, G.G.; LOPES, J. Scarabaeoidea em fragmentos florestais, Londrina, PR. Embrapa Soja. Documentos, 276. p. 86-91, 2006. 
Raul Azevedo; Francisco Roberto de Azevedo; José Antônio de Lima;

Gilberto Barbosa Oliveira e Silva;

Ricardo Braga de Farias

LARSEN, T., LOPERA, A.; FORSYTH, A. Extreme trophic and habitat specialization by Peruvian dung beetles (Coleoptera: Scarabaeidae: Scarabaeinae). The Coleopterists Bulletin, v.60, n.4, p.315-324, 2006.

LESCHEN, R.A.B; MARRIS, J.W.M. Carpophilus (Coleoptera: Nitidulidae) of New Zealand with notes on Australian species. 2012. Disponível em: $<$ http://www.biosecurity.govt.nz/files/regs/exports/plants/ carpophilus.pdf>. Acesso em: 3 de maio de 2012.

LOPES, C.T.; VASCONCELOS, H.L. Evaluation of three methods for sampling ground-dwelling ants in the Brazilian cerrado. Neotropical Entomology, v. 37 n. 4, p. 399-405, 2008.

MARCHIORI, C.H.; De OLIVEIRA, A.T.; LINHARES, A.X. Levantamento de Coleoptera (Arthropoda: Insecta) associados a fezes de gado bovino, no município de Itumbiara, Goiás, Brasil. Acta Scientiarum, v.22, n.2, p.403-407, 2000.

MAGURRAN, A. E. Measuring biological diversity. Malden: Blackwell, 256p. 2004.

MARINONI, R.C.; GANHO, N.G.; MONNÉ, M.L.; MERMUDES, J.R.M. Hábitos alimentares em Coleoptera (Insecta). Ribeirão Preto: Holos, 63p. 2001.

MEDRI, I.M.; LOPES, J. 2001. Scarabaeidae (Coleoptera) do Parque Estadual Mata dos Godoy e Área de Pastagem, no norte do Paraná, Brasil. Revista Brasileira de Zoologia, v.18, (Sup1):135-141, 2001.

MILHOMEM, M.S.; DE MELLO, F.Z.V.; DINIZ, I.R. Técnicas de coleta de besouros copronecrófagos no Cerrado. Pesquisa Agropecuária Brasileira, v.38, n.11, p.1249-1256, 2003.

MOLDENKE, A.R. Arthropods. In: WEAVER, R.W.; ANGLE, S.; BOTTOMLEY, P.; BEZDICEK, D.; SMITH, S.; TABATABAI, A.; WOLLUM, A. Methods of soil analysis: microbiological and biochemical properties. Madison: SSSA, Part 2.,p. 517-542. 1994

MOREIRA, F.M.S.; HUISING, E.J.; BIGNELL, D.E. O inventário da diversidade biológica do solo: conceitos e orientações gerais. In: SWIFT, M.J.; BIGNELL, D.E.; MOREIRA, F.M.S.; HUISING, E.J. Manual de biologia dos solos tropicais. Lavras: UFLA., p.23-39. 2010.

NOGUEIRA-PARANHOS, J.D.; CARVALHO, L.S.; LIMA, M.S.C.S. Métodos de sistemática zoológica. Teresina: EDUFPI/CEAD, 270p. 2015.

OLIVEIRA-COSTA, J. Entomologia forense: quando os insetos são vestígios. 2. ed. Campinas: Millennium, 513p. 2007.

PANIGALLI, G.; SOLIGO, K.T. Diversidade de insecta (arthropoda) associada à carcaça de Sus scrofa L. em um fragmento de Mata Atlântica de Xanxerê, Santa Catarina. Unoesc \& Ciência - ACBS, Joaçaba, v.4, n.1, p.15-26, 2013.

Ciência e Sustentabilidade - CeS / Juazeiro do Norte, v. 4, n. 1, p. 22-38, jan/jun - 2018 
PARR, C.L.; CHOWN, S.L. Inventory and bioindicator sampling: testing pitfall and Winkler methods with ants in a South African savanna. Journal of Insect Conservation, v. 5, p. 27-36, 2001.

PEIXOTO, T.S.; PRAXEDES, C.L.; BACCARO, F.B.; BARBOSA, R.I.; MOURÃO JÚNIOR, M. Composição e riqueza de formigas (Hymenoptera: Formicidae) em savana e ambientes associados de Roraima. Revista Agro@mbiente, v. 4, n.1, p. 1-10, 2010.

QUINTEIRO, T.; LOPES, J.; MARTINS, I.C.F. Diversidade de Carabidae (Coleoptera) amostrados em áreas de reflorestamento de mata ciliar e fragmento florestal, no Estado do Paraná. EntomoBrasilis, v.5, n.5, p.217-222, 2012.

ROCHA, J.R.M. Ocorrência e dinâmica populacional de Scolytidae, Bostrichidae e Platypodidae em povoamentos de eucaliptos e fragmento de cerrado, no município de Cuiabá-MT. 2010. 63f. Dissertação (Mestrado) Universidade Federal de Mato Grosso, Cuiabá. 2010.

SARMIENTO, M.C.E. Metodologías de captura y estudio de las hormigas. In: Fernández, F. (Ed). Introducción a las hormigas de la región Neotropical. Bogotá, Instituto de investigación de Recursos Biológicos Alexander Von Humbolt. 2003. p. 201-210.

SCHOWALTER, T.D. Insect Ecology: an ecosystem approach. Burlington, MA: Academic Press, 633p. 2006.

SILVA, L.N.; Do AMARAL, A.A. Amostragem da mesofauna e macrofauna de solo com armadilha de queda. Revista Verde, v.8, n.5, p.108 - 115, 2013.

SILVA, P.G.; AUDINO, L.D. Escarabeíneos (Coleoptera: Scarabaeidae) atraídos a diferentes iscas em campo nativo de Bagé, Rio Grande do Sul, Brasil. Revista Brasileira de Zoociências, v.13, n 1-3, p.241-247, 2011.

SILVEIRA NETO, S.; NAKANO, O.; BARDIN, D.; VILA NOVA, N.A. Manual de ecologia dos insetos. São Paulo: Agronômica Ceres, 420p. 1976.

SILVEIRA, L.F.; BEISIEGEL, B.M.; CURCIO, F.F.; VALDUJO, P.H.; DIXO, M.; VERDADE, V.K.; MATTOX, G.M.T.; CUNNINGHAM, P.T.M. Para que servem os inventários de fauna? Estudos Avançados, v.24, n.68, p. 173-207, 2010.

SOUZA, J.L.P.; BACCARO, F.B.; LANDEIRO, V.L.; FRANKLIN, E.; MAGNUSSON, W.E. Trade-offs between complementarity and redundancy in the use of different sampling techniques for ground-dwelling ant assemblages. Applied Soil Ecology, v.56, p. 63-73, 2012. 
Raul Azevedo; Francisco Roberto de Azevedo; José Antônio de Lima;

Gilberto Barbosa Oliveira e Silva;

Ricardo Braga de Farias

SPERBER, C.F. Por que há mais espécies de grilo (Orthoptera: Grylloidea) em fragmentos florestais maiores? 1999. 295p. Dissertação de Mestrado.

Universidade Estadual Paulista, Rio Claro.1999.

SPERBER, C.F.; SOARES, L.G.S.; PEREIRA, M.R. Litter disturbance and trap spatial positioning affects number of captured individuals and genera of crickets (Orthoptera: Grylloidea). Journal of Orthoptera Research, v.16, p.1-7, 2007.

TRIPLEHORN, C.A.; JOHNSON, N.F. Estudo dos insetos. São Paulo: Cengage Learning, 2011. 816p.

VALE JÚNIOR, J.F.; LIMA, A.C.S.; CIDADE, M.P.N.; BANDEIRA, H.F.S.; CRUZ, D.L.S. Composição da assembleia de formigas em área de savana no norte da Amazônia. Revista Agro@mbiente, v.11,n.2, p.153-162, 2017.

WINK, C.; GUEDES, J.V.C.; FAGUNDES, C.K.; ROVEDDER, A.P. Insetos edáficos como indicadores da qualidade ambiental. Revista de Ciências Agroveterinárias, v.4, p.60-71, 2005. 